\title{
Section of Photography and Microscopy.
}

Stated Meeting, held Thursday, March 6, 1902.

A PHOTO-MICROGRAPHIC DEVICE.

BY FRLDERIC E. IVES,

Member of the Institute.

Photography, as a means of recording scientific phenomena, is more used and appreciated every day, and would no doubt be more used than it is if all investigators were fully acquainted with its capabilities and equipped to obtain satisfactory results without unnecessary expenditure of time and labor.

Take, for example, the production of photo-micrographs. Probably most people who use a microscope would like occasionally to fix the image which they see; yet comparatively few do so, because it is generally believed that successful photo-micrography involves the exercise of exceptional skill and experience, special and expensive camera devices, rearrangement of microscope and illumination, in positions comparatively difficult to accommodate one's self to, and much time lost. Undoubtedly there is much truth in this, as photo-micrographs are generally made. It is true that a very small and light camera can be attached to the tube of the microscope, over the eye-piece; but under these conditions the amplification in the photographs is much less than it appears in the microscope, and the optical conditions are not of the best.

After making a series of photo-micrographs in the usual way, with the microscope on a special stand and the tube disposed horizontally, I recently set myself the problem of providing for the reproduction of the image observed in the ordinary use of the microscope, without any readjustment whatever, without touching the microscope or even re-focusing. I made the conditions that nothing should interfere in the slightest degree with the comtortable use of the microscope in the usual way; that it might be used 
at any desired inclination or tube-length, and with any convenient source of light; that the adaptation and removal of the camera should occupy very little time, and that the amplification in the photograph should always correspond to that in the microscope.

After some study I set to work and managed, with a boxlid, two small shelf-brackets, the bed and rack of an old $4 \times 5$ camera, a box originally made to carry some $2 \frac{1}{2} \times 3$ plate-holders, a ro-inch focus lens, and some minor odds and ends, to produce the device which I now bring to your notice, and which fulfils all of the conditions I have named.

To commence with, we have the box-lid, a clamped piece of $\frac{1}{2}$-inch mahogany, about Io inches wide and 12 inches long. The microscope is used on this as a base, on which it is held in place by stops against which it is pushed. This base is necessary to provide a fixed support for the camera attachment without touching the microscope itself. Next we have one of the small shelf-brackets, securely attached to the baseboard on each side of the microscope, in such manner that one of the screw-holes in the end comes exactly opposite the center of the joint of the microscope. They are separated just sufficiently to clear all the working parts of the microscope. The screw-holes in the brackets are the points of attachment for the camera device, and must occupy such a position in order that the camera may swing from the same center as the microscope body, and thus be adjustable by a single movement for any desired inclination, from horizontal to vertical. On a double pillar microscope, and even on the Swift portable which I am using, the camera attachment could be adapted to swing from the centers on the microscope itself, and the special base and brackets thus dispensed with; but with the usual horseshoe base "continental" stands the bracket supports are necessary. The total height of the bracket supports for a Bausch \& Lomb "BB" stand is not over $3 \frac{1}{2}$ inches, and they are not in the least in the way of anything one ever wants to do with a microscope. A detachable double upright can, however, be used, if preferred, and fastened to the microscope base at a moment's notice. 
The camera is a simple box, with a lens at one end and fitting for a plate-holder at the other-the lens of ro-inch focus, and the distance from the lens to the sensitive plate, ro inches. This length of camera insures an image having the same amplification that would be calculated for that in the microscope, provided that its lens occupies the normal position of the eye above the eye-piece. The ro-inch camera and lens are used on the assumption that the position of the objective and eye-piece cannot be altered without altering the character of the image; that the rays forming each pencil of light emerging from the eye-piece are parallel to each other when the microscope is focussed, and that the camera must, therefore, focus parallel rays in order to make a sharp image of the microscope projection at the focal plane without re-focusing the microscope. This assumption is correct under certain conditions, which I shall specify later.

I did not have a Io-inch focus lens, but had $\mathrm{z}$-inch and 6o.inch plano-convex lenses. A pair of these cemented together made a crossed lens of about io.inch focus, and I mounted it in an old view-lens tube, so that the flattest side comes towards the eye-piece, but an inch back from the eyepoint, when the mounts almost touch each other. I should not have used this lens mount, because, although it does not affect the focus upon the ground-glass to move the lens back from the eye-point, it does alter the size of the image by bending the outer pencils of the cone of light in towards the middle. One should either use a double-corrvex lens at the eye-point, or a lens of longer focus above the eye-point, in combination with a correspondingly longer camera, in order to keep the amplification strictly correct.

On general principles, I would not select a non-achromatic lens for this purpose, and do not recommend it ; but it happens that with some achromatic objectives and huygenian eye-pieces a non-achromatic lens gives the best projection. It does not make much difference whether it be achromatic or not if monochromatic light is used for photographing, which is what I recommend with this method of working. The fact that objective and eye-piece combinations do vary 
somewhat in this respect makes it a safe rule to photograph with approximately monochromatic light of the brightest part of the spectrum, thereby insuring definition equal to that seen in the microscope.

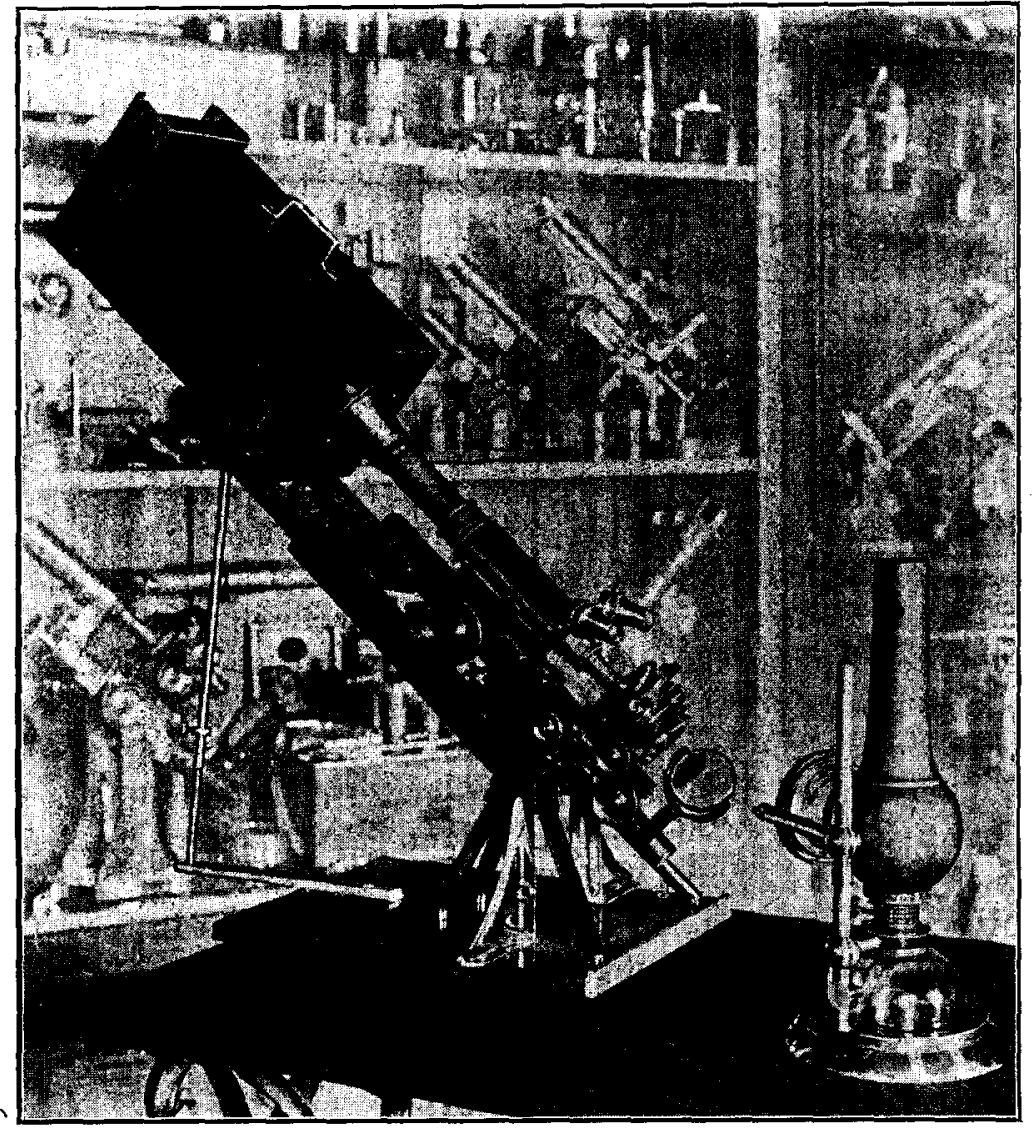

Photo-micrographic camera adapted to a Swift Folding microscope, used on a special base with brackets.

The camera has a rack and pinion movement on a baseboard having two rigidly attached arms extending forward, and carrying pins to engage in the screw-holes of the brackets. I have cut slots into the screw-holes, so that the pins drop into place, and provided an automatic lock which 
prevents the pins from being lifted out except when the camera is swung below the horizontal plane. An adjustable telescopic strut and detachable extension to the baseboard supports the camera and fixes it at any desired inclination. It is in alignment with microscope tube when the camera lens-mount and the eye-piece are concentric with

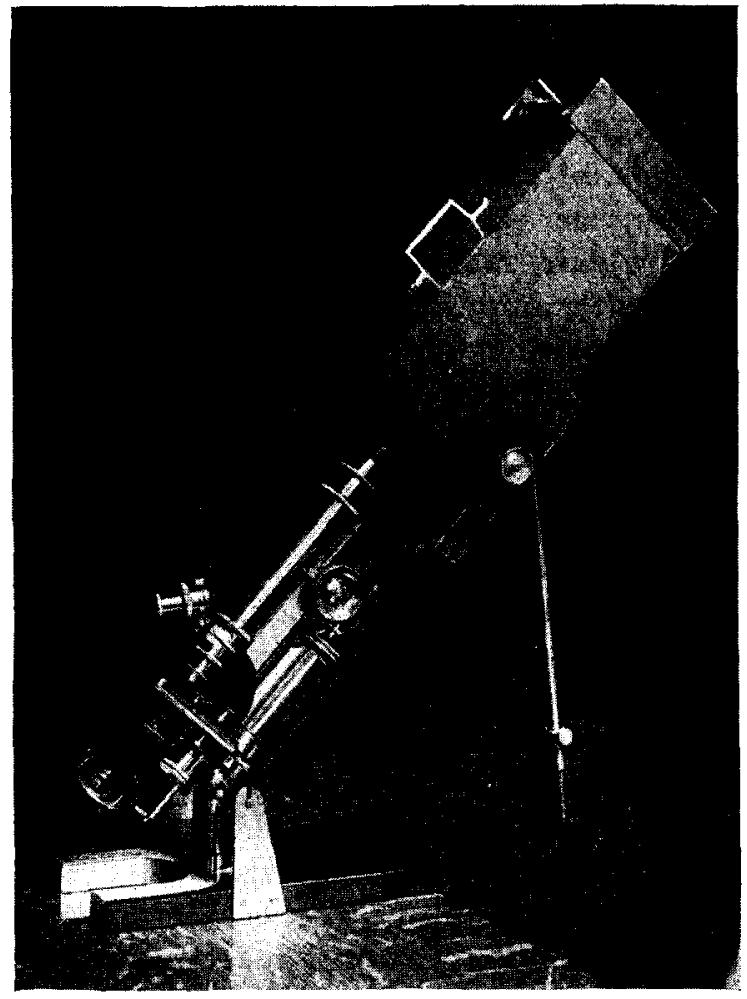

Photo-micrographic camera adapted to a Bausch \& Lomb " BB" micrcscope, with detachable bracket support.

each other when brought together by the rackwork. For optical reasons, strict alignment is far less important than it would be with a camera containing no lens.

It takes me from twenty to thirty seconds to attach the camera and adjust ready for an exposure. A color screen is then [usually] placed between the source of light and the 
microscope mirror, admitting approximately monochromatic light of such color as the objectives are best corrected for. Plate-holder is then inserted, and exposure made, using an isochromatic plate.

Photo-micrographs made in this way, ranging from comparatively low amplifications $u p$ to $\mathrm{I}, 500$ diameters, cannot be distinguished in character or quality from similar ones which were made after most careful preparation with far more elaborate, expensive and troublesome photo-micrographic apparatus.

Heretofore, I have done this work on a specially heavy table, legs resting upon rubber buffers, heavy marble top also resting on rubber buffers, an inch-board resting on coils of rubber tubing. Without these precautions, I had trouble with vibration when the microscope was used in a horizontal position. I have been using the new device on an ordinary table, and have been surprised to find no difficulty whatever with vibration, so far. The microscope appears to be far less sensitive when inclined at approximately $45^{\circ}$ angle than when either horizontal or vertical. I exposed two hours on an amphipleura with oblique light from a Welsbach burner, and the lines came out sharp. Most subjects, with central light, require only one to five minutes' exposure, using rapid isochromatic plates.

I have stated that one of my objects was to avoid the necessity of re-focusing the image, which is objectionable, both because of the time it takes and because it alters, however slightly, the character of the image. The conditions under which no re-focusing is necessary are, that the camera lens shall focus parallel rays at the focal plane of the camera, and that the microscope shall have been focussed with an eye that focuses pefectly parallel rays upon its retina. Under these conditions, the rays forming each pencil of light emerging from the eye-piece are parallel to each other, and will therefore come to a perfect focus at the focal plane of the camera. If the microscope were focussed by a short-sighted or an abnormally far-sighted eye, the rays forming the emerging pencils would not be parallel to each 
other, and therefore would not come to a perfect focus at the focal plane of the camera.

Huygenian eye-pieces, and some others, are calculated for an eye that focuses parallel rays, and cannot give the best results to either a short-sighted or an abnormally far-sighted eye. A short-sighted person sees an image which is formed above the correct plane in the eye-piece, and an abnormally far-sighted person sees an image which is formed below the correct plane. A person having theoretically normal sight will focus the microscope correctly, not only for the best image obtainable, but for the focal plans of my photo-micrographic camera. Any one whose eye is not theoretically normal will have to re-focus on the ground-glass of the camera when using low powers; but when he has done so, the image will be exactly what it should be. My own eyes are short-sighted, and in pursuit of my object I have as nearly as possible overcome this difficulty by providing myself with a compensating lens in a mount which fits loosely over the eye-pieces of the microscope, and makes my vision, for the time being, theoretically normal. As this lens belongs to my eye, and not to the eye-piece, I remove it after focusing. This procedure is equivalent to wearing spectacles adjusted for clearest vision at very great distances, which any one may do; but the eyes of very young people, with great powers of accommodation, may not even then be trusted implicitly when working with very low powers. With high-power objectives and eye-pieces, hypermetropes, emmetropes and moderate myopes focus practically all alike.

This new outfit is not calculated to supersede some of the very elaborate and expensive photo-micrographic apparatus and devices which have been produced by Zeiss and others for some special purposes. It will not produce photographs of large size, such as can be made with projection eye-pieces and very long cameras, nor include wide angles of view in low powers, such as may be obtained with Zeiss planar lenses without eye-pieces; but it should be far more generally useful, because it can be relied upon to reproduce clearly whatever detail the eye sees in the microscope, and 
with the least possible cost, either in time, labor or expenditure for equipment.

It will be evident that the same camera and procedure as to focusing is adapted to making records with telescopes, spectroscopes and some other optical instruments.

\section{ELECTRICITY DIRECT FROM COAL.}

The latest of the many indefatigable attempts made to obtain electricity direct from coal is that of Hugo Johe, of Chicago. He has obtained the patent on a new battery. In his invention a retort is employed, which is surrounded by a cylindrical case containing a battery of four cells. The furnace gases from the retort circulate against the inner side of the cell and case.

Partitions of porous coal divided each cell into three sections, containing respectively nitric acid, sulphuric acid and ferric chloride solution. In the nitric acid an electrode is immersed, and in the narrow chloride solution a lead electrode.

The operations are as follows: A suitable quantity of sulphate of lead is placed in the retort with a quantity of coal nearly sufficient to reduce the sulphate to sulphide, and the mixture is then heated until all of the coal is oxidized. The sulphide is freed from impurities which may have been brought into it by the coal, and is then mixed with sulphate of lead in suffcient quantity to yield metallic lead and sulphur dioxide, which reduction is effected by again applying fuel heat to the retort. The sulphur dioxide passes through a pipe into the larger section of the several cells, reducing the ferric chloride therein to ferrous chloride. Previous to this the generation of the electric current is started by putting the lead electrodes into the larger section and suitably connecting them with the carbon electrodes. The current may be considered as consisting of two currents, one generated by the action of the ferric chloride of the lead electrode, and the other by the action of the nitric acid through the interposed porous walls, and sulphuric acid by means of molecular exchange of ferrous chloride. The flow of sulphur dioxide is so regulated that the sulphuric acid formed is not more than sufficient to decompose the chloride of lead formed in the battery reaction. The lead in the retort is allowed to flow into a pan, where it'is suitably shaped or solidified for an electrode. The sulphate of lead deposited by the battery is allowed to accumulate, and at intervals is drawn off by means of siphons, and the deposit of sulphate of lead electrode removed. The temperature of the battery is regulated so that the nitric acid which enters into the sulphuric section is evaporated, the vapors being passed through a condenser and there condensed again to nitric acid, flowing back into the nitric acid sections through a pipe. This process of distillation, oxidation and condensation is kept up by regulating the temperature of the battery and supplying sufficient cooling water to the condenser. Thus oxygen is supplied to the nitric acid, while the generation of electric energy with consumption of oxygen goes on. The E.M.F. of the cell at $100^{\circ} \mathrm{C}$. is said to be about $I^{\circ} 75$ volts.-Scientific American Supplement. 